\title{
Correction: Strongly correlated plexcitonics: evolution of the Fano resonance in the presence of Kondo correlations
}

$2015,17,14238$

A. Goker

DOI: $10.1039 / c 5 c p 90078 f$

Correction for 'Strongly correlated plexcitonics: evolution of the Fano resonance in the presence of Kondo correlations' by A. Goker et al., Phys. Chem. Chem. Phys., 2015, 17, 11569-11576.

www.rsc.org/pccp

(a) The authors would like to correct a couple of typos in eqn (8) and (9) on page 11571. The last $G_{\mathrm{e}}^{>}\left(t, t^{\prime}\right)$ in both equations should be replaced by $B_{\mathrm{e}}^{>}\left(t, t^{\prime}\right)$. Therefore, eqn (8) and (9) should be as follows:

$$
\xi_{\mathrm{pe}}^{<}\left(t, t^{\prime}\right)=|\Delta|^{2} G_{\mathrm{e}}^{<}\left(t, t^{\prime}\right) B_{\mathrm{g}}^{<}\left(t, t^{\prime}\right) G_{\mathrm{g}}^{>}\left(t, t^{\prime}\right) B_{\mathrm{e}}^{>}\left(t, t^{\prime}\right)
$$

and

$$
\xi_{\mathrm{pe}}^{>}\left(t, t^{\prime}\right)=|\Delta|^{2} G_{\mathrm{e}}^{>}\left(t, t^{\prime}\right) B_{\mathrm{g}}^{>}\left(t, t^{\prime}\right) G_{\mathrm{g}}^{<}\left(t, t^{\prime}\right) B_{\mathrm{e}}^{<}\left(t, t^{\prime}\right) .
$$

(b) In Fig. 3, 4 and 6, the captions incorrectly refer to a purple solid line representing the data at $T=24 \mathrm{~K}$. This should refer to a green solid line instead.

(c) $c_{\mathrm{g}(\mathrm{e}) \sigma}$ in the second line in eqn (2) should be $c_{\mathrm{g}(\mathrm{e}) \sigma}^{\dagger}$ instead.

(d) The word "less" in the sentence before eqn (6) should be "greater" instead. Eqn (7) refers to the greater than Green function clearly.

(e) The word "and" in the sentence before eqn (14) should be "which" instead.

The Royal Society of Chemistry apologises for these errors and any consequent inconvenience to authors and readers. 